\title{
The influence of size effects on local piezoelectric response of thin films
}

\author{
A.N. Morozovska, S.V. Svechnikov \\ V. Lashkaryov Institute of Semiconductor Physics, National Academy of Science of Ukraine \\ 41, prospect Nauky,03028 Kyiv, Ukraine, e-mail:morozo@i.com.ua
}

\begin{abstract}
We discuss the influence of size effects on the local piezoelectric response of thin films. In calculations of the electrostatic potential in the triple system "PFM probe tip film - substrate," the effective point charge model is used. The obtained expressions for the local piezoelectric response of a surface layer (film) capped are intended for the calculations of Piezoresponse Force Microscopy signals of thin polar films epitaxially grown on thick substrates. Theoretical predictions are in qualitative agreement with typical experimental results obtained for perovskite $\mathrm{Pb}(\mathrm{Zr}, \mathrm{Ti}) \mathrm{O}_{3}$ and multiferroic $\mathrm{BiFeO}_{3}$ films.
\end{abstract}

Keywords: local piezoelectric response, size effects.

Manuscript received 14.06.07; accepted for publication 19.12.07; published online 31.01.08.

\section{Introduction}

It is well recognized that film piezoelectric and dielectric properties can be strongly modified as compared with those in bulk. For instance, the surfaces of nonpiezoelectric materials can reveal a built-in dipole moment due to the inversion symmetry breaking and thus possess surface piezo- and flexoelectricity. In centrosymmetric materials, symmetry breaking at surfaces and interfaces can give rise to surface piezoelectric coupling even in non-polar materials [1].

Several theoretical groups analyzed the effect of properties of the films of polar materials and hysteresis loop features within the framework of the Landau-GinzburgDevonshire phenomenology. (see, e.g., Refs [2-9]).

For the verification of theoretical models, elaboration of functional nanomaterials with predetermined properties, and application in various devices (such as ferroelectric micro- and nanocapacitors, sensors, actuators, etc.), the recognition and diagnostics of piezoelectric films and their multilayer structures are rather important. In thin films, the vertical shift of piezoelectric and ferroelectric hysteresis loops was interpreted in terms of a non-switchable layer by Saya et al. [2]. Alexe et al. [3] analyzed the hysteresis loop shape in ferroelectric nanocapacitors with top electrode and obtained the estimation for a switchable volume. A similar analysis was applied to ferroelectric nanoparticles developed by the self-patterning method [4] by $\mathrm{Ma}$ [5]. In all cases, the results were interpreted in terms of 2 20 nm non-switchable layers, presumably at the ferroelectric-electrode interface.

Recently, the development of Piezoresponse Force Microscopy (PFM) has allowed the 2D mapping of the switching behavior in piezoelectric thin films. However, the existing framework for the data analysis is invariably based on the 1D models suggested originally by Ganpule, thus ignoring the 3D geometry of the PFM problem. Recently, we have applied [6] the decoupled theory [7, 8] to derive analytical expressions for the PFM response on semi-infinite low-symmetry materials. However, to the best of our knowledge, there are no analytical results of calculations of the effective piezoelectric response of thin piezoelectric films epitaxially grown on thick substrates.

Here, we analyze the local effective piezoresponse (PFM signal) of a piezoelectric film using the decoupling approximation. The obtained results can be successfully used for calculations of the effective piezoelectric response in the case of a ferroelectric or piezoelectric film capped on the nonpiezoelectric substrate with close elastic properties (like $\mathrm{PbTiO}_{3}$ or $\mathrm{BaTiO}_{3}$ on $\mathrm{SrTiO}_{3}$ or $\mathrm{SrRuO}_{3}$ ).

\section{Problem}

\subsection{Decoupling approximation}

The PFM signal, e.g., the surface vertical displacement $u_{3}(\mathbf{x}, \mathbf{y})$ at a point $\mathbf{x}$ induced by the tip at the position $\mathbf{y}=\left(y_{1}, y_{2}\right)$, is given by 


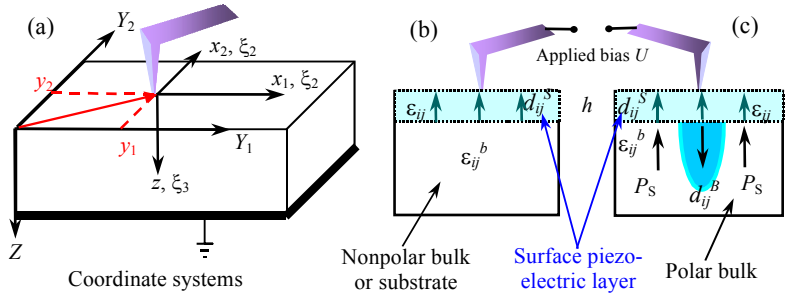

Fig. 1. (a) Coordinate systems in the PFM experiment. (b, c) structure of the considered systems: (b) the piezoelectric layer (film) capped on a nonpolar bulk (substrate) with the same elastic properties; (c) unswitchable piezoelectric layer capped on a polar bulk.

$$
\begin{aligned}
& u_{3}(\mathbf{x}, \mathbf{y})=\int_{-\infty}^{\infty} d \xi_{1} \int_{-\infty}^{\infty} d \xi_{2} \int_{0}^{\infty} d \xi_{3} \frac{\partial G_{3 j}^{f}\left(x_{1}-\xi_{1}, x_{2}-\xi_{2}, \xi_{3}\right)}{\partial \xi_{k}} \times \\
& \times E_{l}(\xi) c_{k j m n} d_{l n m}\left(y_{1}+\xi_{1}, y_{2}+\xi_{2}, \xi_{3}\right) .
\end{aligned}
$$

Here, the coordinate $\mathbf{x}=\left(x_{1}, x_{2}, x_{3}\right)$ is linked to the tip apex, and the coordinates $\mathbf{y}=\left(y_{1}, y_{2}\right)$ denote the tip position in the sample coordinate system $\mathbf{y}$ (see Fig. 1a); the coefficients $d_{m n k}$ and $c_{j l m n}$ are components of the piezoelectric strain constant and elastic stiffness tensors, respectively; $E_{k}(\mathbf{x})=-\partial \varphi_{i}(\mathbf{x}) / \partial x_{k}$ is the $a c$ electric field distribution produced by the probe $\left(\varphi_{i}\right.$ is its potential); and the Green's function for a film, $G_{3 j}^{f}(\mathbf{x}, \xi)$, is derived in Ref. [9] and depends on mechanical boundary conditions on the film-substrate interface. For the surface piezoelectric layer appeared on the non-polar bulk, as well as for the films on substrates with matched elastic properties, one can use the Green function $G_{3 j}^{S}$ of a semiinfinite medium given in Ref. [6].

\subsection{Electrostatic potential}

In the case of a dielectrically transversely isotropic piezoelectric film, the inner electrostatic potential $\varphi_{i}$ created by a point charge $Q$ located at distance $d$ outside the layer has the following form:
Here, $\sqrt{x_{1}^{2}+x_{2}^{2}}=\rho$ and $x_{3}$ are the radial and vertical coordinates, respectively, $J_{0}(x)$ is the zeroorder Bessel function, $\varepsilon_{e}$ is the dielectric constant of the ambient, $\kappa=\sqrt{\varepsilon_{33} \varepsilon_{11}}$ is the effective dielectric constant, $\gamma=\sqrt{\varepsilon_{33} / \varepsilon_{11}}$ is the dielectric anisotropy factor of the film, and $\kappa_{b}=\sqrt{\varepsilon_{33}^{b} \varepsilon_{11}^{b}}$ is the effective dielectric constant of the bulk. Since the electrostatic potential is a linear function of the applied electric field, the point charge potential (2) provides the basic model, and the results for realistic tip geometries can be obtained using an appropriate image charge model with the help of the additional summation or integration over the set of real or image charges representing the tip. Unfortunately, in the considered case of two boundaries (the triple system "ambient - dielectric 1 - dielectric 2"), the image charge method is not suitable.

An alternative approach to the description of electric fields in the immediate vicinity of the tip-surface junction is the effective point charge model $[10,11]$, in which the charge value $Q$ and its surface separation $d$ are selected so that the corresponding isopotential surface reproduces the tip radius of curvature $R_{0}$ and potential $U$. We succeed to evolve the effective point charge approach for the spherical tip potential corresponding to the considered triple system "ambient - film - bulk" and obtained the exact series in image charges for the determination of $Q$ and $d$. For the tip that touches the surface, their Pade approximations have the form:

$d \approx d_{\infty} \frac{\kappa_{b} h^{2}+\kappa \gamma^{2} R_{0}^{2}}{\kappa_{b}\left(\gamma^{2} R_{0}^{2}+h^{2}\right)}$

$Q(h, d) \approx Q_{\infty}\left(1+\left(\frac{\kappa_{b}+\varepsilon_{e}}{\kappa-\kappa_{b}}+\frac{h}{\gamma d} \frac{\varepsilon_{e}-\kappa}{\kappa} \times\right.\right.$

$\left.\left.\times \ln ^{-1}\left(1-\frac{\kappa_{b}-\kappa}{\kappa_{b}+\kappa} \cdot \frac{\varepsilon_{e}-\kappa}{\varepsilon_{e}+\kappa}\right)\right)^{-1}\right)^{-1}$.

Saturation values $\quad d_{\infty}=\varepsilon_{e} R_{0} / \kappa \quad$ and $Q_{\infty}=2 \pi \varepsilon_{0} \varepsilon_{e} R_{0} U\left(\kappa+\varepsilon_{e}\right) / \kappa \quad$ correspond to the semiinfinite system "ambient-dielectric" [10].

The dependences of the effective charge surface separation $d$ and its value $Q$ on the layer thickness $h$ are shown in Fig. 2 for both the relatively low dielectric permittivity $\kappa=30(\mathrm{a}, \mathrm{c})$ and high dielectric permittivity $\kappa=3000(b, d)$ of the film.

$$
\varphi_{i}\left(\rho, x_{3}\right)=\frac{Q}{2 \pi \varepsilon_{0}} \int_{0}^{\infty} d k J_{0}(k \rho) \frac{\left(\kappa_{b}+\kappa\right) \exp \left(-k d-k \frac{x_{3}}{\gamma}\right)-\left(\kappa_{b}-\kappa\right) \exp \left(-k d-k \frac{2 h-x_{3}}{\gamma}\right)}{\left(\kappa_{b}+\kappa\right)\left(\varepsilon_{e}+\kappa\right)-\left(\kappa_{b}-\kappa\right)\left(\varepsilon_{e}-\kappa\right) \exp \left(-\frac{2 h}{\gamma} k\right)} .
$$



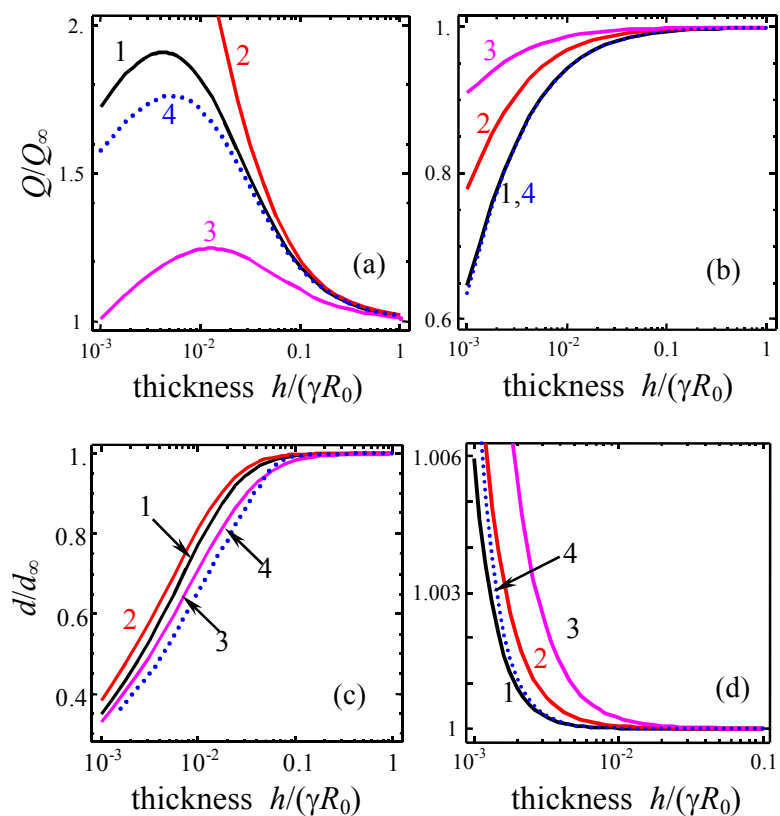

Fig. 2. Dependence of the effective charge $Q / Q_{\infty} \quad(\mathrm{a}, \mathrm{b})$ and $d / d_{\infty}$ (c, d) on the surface layer thickness with dielectric permittivity $\kappa=30,3000 \quad[(\mathrm{a}, \mathrm{c})$ and $(\mathrm{b}, \mathrm{d})$, respectively]. Other parameters: dielectric anisotropy $\gamma=1$, ambient dielectric constant $\varepsilon_{e}=1$, and bulk dielectric constant $\kappa_{b}=260$. Curves 1, 2, 3 were calculated for the systems of 50, 4, and 2 image charges. Dotted curves 4 represent approximations (3).

Under the condition $h \geq \gamma R_{0}$, the values $d \rightarrow d_{\infty}$ and $Q \rightarrow Q_{\infty}$. Since the dielectric anisotropy $\gamma$ is less that unity $(0<\gamma \leq 1)$ for the majority of perovskites and the dielectric constant $\kappa>10$ for the majority of polar materials, our calculations essentially improve the heuristic condition imposed on the layer thickness, $h>>\gamma R_{0}$, for the semiinfinite approximation to be valid in films. Namely, when the piezoelectric film thickness $h$ is close or more than the tip curvature $R_{0}\left(h \geq R_{0}\right)$, the substrate (or bulk) does not affect the surface electrostatic potential $\varphi_{i}\left(x_{3}=0\right)$ almost independently on the ratio of film/substrate dielectric permittivities.

\subsection{Piezoelectric properties}

Here, we consider the case where the dielectric and piezoelectric properties of a layer (or film) differ from those of the bulk or substrate. In this case, the strain piezoelectric coefficient $d_{k l j}\left(y_{1}, y_{2}, x_{3}\right)$ is dependent on the depth $x_{3}$ as follows:

$d_{k l j}\left(y_{1}, y_{2}, x_{3}\right)=\left\{\begin{array}{l}d_{i j k}^{S}\left(y_{1}, y_{2}\right), \quad 0 \leq x_{3} \leq h \\ d_{i j k}^{B}\left(y_{1}, y_{2}, x_{3}\right) \quad \text { or } 0, h<x_{3}<\infty .\end{array}\right.$
Here, $d_{i j k}^{S}\left(y_{1}, y_{2}\right)$ and $d_{i j k}^{B}\left(y_{1}, y_{2}, x_{3}\right)$ are the piezoelectric effect tensors of the film and substrate, respectively. For a non-piezoelectric substrate, $d_{i j k}^{B}\left(y_{1}, y_{2}, x_{3}\right) \equiv 0$ (see Fig. 1b, c).

\section{Effective local piezoelectric response}

\subsection{Homogeneous surface layers and the piezoelectric response of thin films}

Assuming that the piezoelectric coupling is uniform inside the film regions (domains) with transverse sizes much greater than the tip curvature, i.e., $d_{l k j}^{S}\left(y_{1}, y_{2}, x_{3} \leq h\right) \approx$ const at $\sqrt{y_{1}^{2}+y_{2}^{2}}>>R_{0}$, the film vertical piezoresponse $d_{33}^{\text {eff }}=u_{3}(\mathbf{r}=0) / \varphi_{i}(\mathbf{r}=0)$ has the form:

$d_{33}^{\text {eff }}(h, d)=\frac{W_{333}^{f}}{\psi_{i}} d_{33}^{S}+\frac{W_{313}^{f}}{\psi_{i}} d_{31}^{S}+\frac{W_{351}^{f}}{\psi_{i}} d_{15}^{S}$,

where the components $W_{3 i j}^{f}$ are

$W_{333}^{f}(h, d)=-\sum_{m=0}^{\infty}\left(\frac{\kappa_{b}-\kappa}{\kappa_{b}+\kappa}\right)^{m}\left(\frac{\varepsilon_{e}-\kappa}{\varepsilon_{e}+\kappa}\right)^{m}$

$\left(\frac{d}{\gamma d+2 h m}+\frac{\kappa_{b}-\kappa}{\kappa_{b}+\kappa} \frac{d}{\gamma d+2 h(m+1)}\right) \times$

$\times \frac{\gamma h}{(\gamma d+(1+2 m+\gamma) h)}\left(1+\frac{\gamma h}{(\gamma d+(1+2 m+\gamma) h)}\right)$,

$W_{313}^{f}(h, d)=-\sum_{m=0}^{\infty}\left(\frac{\kappa_{b}-\kappa}{\kappa_{b}+\kappa}\right)^{m}\left(\frac{\varepsilon_{e}-\kappa}{\varepsilon_{e}+\kappa}\right)^{m} \times$

$\times\left(\frac{d}{\gamma d+2 h m}+\frac{\kappa_{b}-\kappa}{\kappa_{b}+\kappa} \frac{d}{\gamma d+2 h(m+1)}\right) \times$

$\times \frac{\gamma h}{(\gamma d+(1+2 m+\gamma) h)}\left((1+2 v)-\frac{\gamma h}{(\gamma d+(1+2 m+\gamma) h)}\right)$,

$W_{351}^{f}(h, d)=-\sum_{m=0}^{\infty}\left(\frac{\kappa_{b}-\kappa}{\kappa_{b}+\kappa}\right)^{m}\left(\frac{\varepsilon_{e}-\kappa}{\varepsilon_{e}+\kappa}\right)^{m} \times$

$\times\left(\frac{d}{\gamma d+2 h m}-\frac{\kappa_{b}-\kappa}{\kappa_{b}+\kappa} \frac{d}{\gamma d+2 h(m+1)}\right) \times$

$\times \frac{\gamma^{3} h^{2}}{(\gamma d+(1+2 m+\gamma) h)^{2}}$.

At the same time, $\psi_{i}$ has the form:

$$
\begin{aligned}
& \psi_{i}(h, d)=\sum_{m=0}^{\infty}\left(\frac{\kappa_{b}-\kappa}{\kappa_{b}+\kappa}\right)^{m}\left(\frac{\varepsilon_{e}-\kappa}{\varepsilon_{e}+\kappa}\right)^{m} \times \\
& \times\left(\frac{\gamma d}{\gamma d+2 h m}-\frac{\kappa_{b}-\kappa}{\kappa_{b}+\kappa} \frac{\gamma d}{\gamma d+2 h(m+1)}\right) .
\end{aligned}
$$


We note that the response theorem $u_{3}\left(0,0, x_{3}\right) \sim \varphi_{i}\left(0,0, x_{3}\right)$ formulated in Ref. [6] for semiinfinite piezoelectric materials is not valid for a layer of finite thickness $h$, because the ratio $W_{3 j k}^{f} / \psi_{i}$ is dependent on $h$ and $d$ in accordance with Eqs. (6)-(9). This becomes clear since some image charges are located below the layer (i.e., in the region $x_{3}>h$ ).

When the dielectric permittivity $\kappa$ lies in the interval $\varepsilon_{e}<\kappa<\kappa_{b}$, the first two terms for $m=0$ (point charge + its first image) provide accuracy not less than $5 \%$ even if $\left|\frac{\kappa_{b}-\kappa}{\kappa_{b}+\kappa}\right| \cong 0.95$. For $\kappa>\kappa_{b}$, series

converge more slowly, and the accuracy not less than $10 \%$ corresponds to the case where $\left|\frac{\kappa_{b}-\kappa}{\kappa_{b}+\kappa}\right| \leq 0.5$. Under the condition $h / \gamma d>>1$, i.e., for a thick film, Eq. (10) coincides with the expression for $d_{33}^{\text {eff }}$ obtained earlier in Ref. [6] for a semiinfinite system $d_{33}^{\text {eff }}(h, d) \approx-\frac{\gamma^{2} d_{15}^{S}}{(1+\gamma)^{2}}-\frac{d_{33}^{S}}{(1+\gamma)^{2}}-\left(1-\frac{(1+2 v)}{1+\gamma}\right) d_{31}^{S}$, as it should be expected. For ultra-thin layers $(h<<d)$, Eq. (10) yields $d_{33}^{\text {eff }} \approx-\frac{h}{\gamma d}\left(d_{33}^{S}+(1+2 v) d_{31}^{S}+\frac{\gamma h}{d} d_{15}^{S}\right)$.

\subsection{Size effects of the piezoelectric properties of thin films}

Analyzing the basic results of Section 3.1, we would like to underline once more that they have independent application to calculations of the effective piezoelectric response $d_{33}^{\text {eff }}(h)$ in the case of a ferroelectric or piezoelectric film capped on a non-piezoelectric bulk. Such a situation for the nanostructure $\mathrm{BaTiO}_{3} / \mathrm{SrTiO}_{3}$ is shown in Fig. 3.

As follows from Fig. 3, the condition $h / d \geq 10^{2}$ is sufficient for the effective piezoresponse of a layer to become thickness-independent. As usual, $d \sim 1-100 \mathrm{~nm}$, thus the piezoelectric response appeared thicknessdependent for the thickness of films to be less than $100 \mathrm{~nm}$ (extrinsic size effect). The main reasons of the considered extrinsic size effect are the thicknessdependent structure of elastic strains and the electrostatic potential, as well as the finiteness of the signal generation volume [12].

The extrinsic size effect should be clearly distinguished from the intrinsic size effects related to the inhomogeneous polarization distribution in nanostructures. Moreover, the extrinsic size effect can interfere with several intrinsic ones caused by filmsubstrate misfit strains, a decrease of the correlation volume, and the depolarization field $[13,14]$. For thin

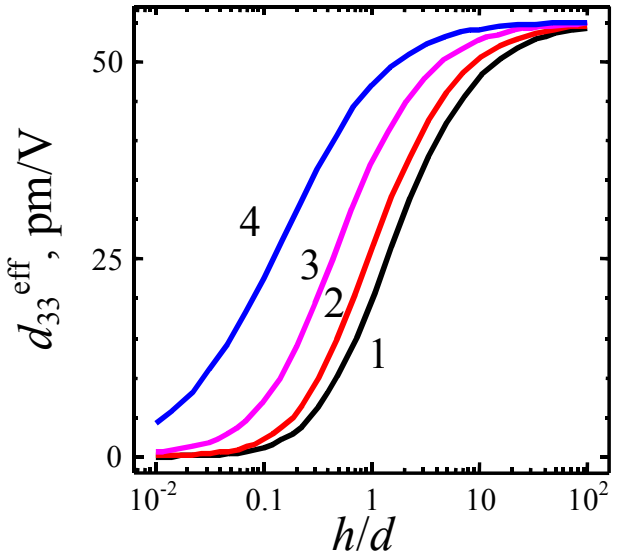

Fig. 3. Effective piezoelectric response $d_{33}^{\text {eff }}(h)$ of $\mathrm{BaTiO}_{3}$ $(\kappa=700, \gamma=0.24)$ film capped on a non-piezoelectric bulk with close elastic properties $(v=3)$, but different dielectric constants $\kappa_{b}=3 ; 30 ; 300 ; 3 \times 10^{3}$ (curves 1, 2, 3, 4, respectively); $\varepsilon_{e}=1$. Note that the scaling $d \rightarrow \varepsilon_{e} R_{0} / \kappa$ for the effective point charge model of a tip is valid under the conditions $0.1<\kappa_{b} / \kappa<10$ and $h \geq 0.2 \gamma R_{0}$.

films, the dielectric permittivity $\varepsilon_{i i}(h)$ and the spontaneous polarization $P_{S}(h)$ are thickness-dependent. Since the piezoelectric constants $d_{i j}^{S} \sim \varepsilon_{i j} P_{3}^{S}$, the dependence $d_{i j}^{S}(h)$ should be included in Eq. (5).

The size-driven phase transition into the paraelectric (non-polar) phase appeared in thin films with thicknesses $h \leq h_{\text {cr }}$, where the critical thickness $h_{\text {cr }}$ depends on temperature, stress, etc. For instance, $h_{\text {cr }}=1-2 \mathrm{~nm}$ for $\mathrm{PbTiO}_{3}$ on $\mathrm{SrTiO}_{3}$ substrate and $h_{\text {cr }} \leq 5 \mathrm{~nm}$ for $\mathrm{BaTiO}_{3}$ on $\mathrm{SrRuO}_{3}$ substrate at room temperature (see Refs. $[15,16]$ for more details). If the values of $h_{\text {cr }}$ and $d$ are of the same order, the extrinsic and intrinsic size effects would interfere. Their contributions can be separated for a homogeneous (single-domain) film by fitting the experimental data provided by Eq. (5) accompanied with appropriate dependence $d_{i j}^{S}(h)$. Such a situation is demonstrated in Fig. 4 for $\mathrm{PbZr}_{0.52} \mathrm{Ti}_{0.48} \mathrm{O}_{3}$ film on $\mathrm{SrTiO}_{3}$ substrate.

\subsection{Vertical shift of the piezoresponse for "surface piezoelectric layer + switchable bulk"}

In the case of an irreversible surface polar layer, the vertical shift $d_{33}^{V}$ caused by the surface piezoeffect should be distinguished from the downward loop shift $d_{33}^{D}$ originated from the onset of a nested domain inside the existed one and its domain wall pinning (bulk polarization switching in the kinetic limit) considered in details in Refs. [10, 17]. However, the vertical asymmetry $d_{33}^{D}\left(U_{\max }\right)$ considered by the onset of a 

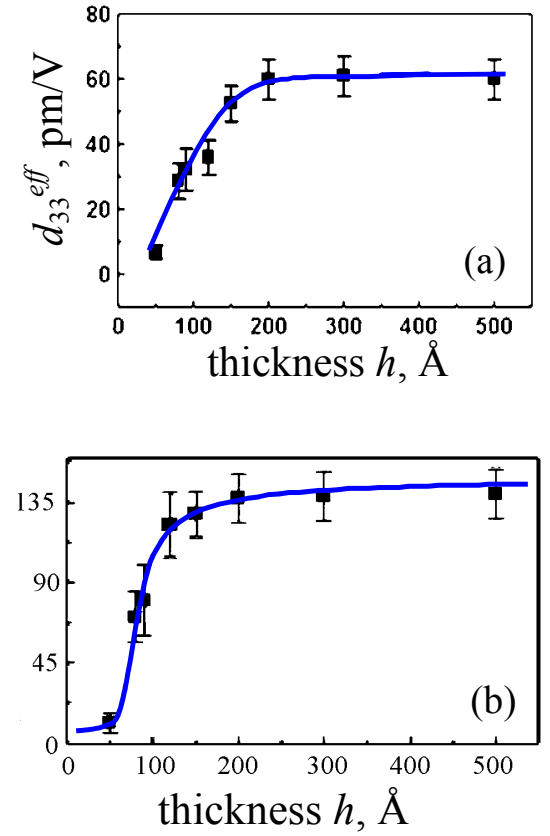

Fig. 4. (a) Effective piezoelectric response $d_{33}^{\text {eff }}(h)$ versus the thickness of a $\mathrm{PbZr}_{0.52} \mathrm{Ti}_{0.48} \mathrm{O}_{3}$ film on $\mathrm{SrTiO}_{3}$ substrate. Symbols are the experimental data from Ref. [14], solid curve is the theoretical fitting with the help of Eq. (5) at $d=2 \mathrm{~nm}$ allowing for the dependence (b) for polarization $P_{S}(h)$. The dependence for was fitted by the formula $P_{S}(h) \approx P_{i}+P_{S}^{b} \sqrt{1-h_{\mathrm{cr}} / h}$, where the bulk polarization $P_{S}^{b}=135 \mu \mathrm{C} / \mathrm{cm}^{2}$ and the surface polarization $P_{i} \approx$ $5 \mu \mathrm{C} / \mathrm{cm}^{2}$ (appeared from symmetry breaking) and the critical thickness $h_{\mathrm{cr}}=5 \mathrm{~nm}$.

nested domain inside the existed one depends on the maximal $d c$ bias $U_{\max }$ applied to the probe (i.e., on the domain size) and decreases with increase in $U_{\max }$, whereas the shift $d_{33}^{V}=d_{33}^{\text {eff }}$ described by Eq. (5) is voltage-independent, but depends on the layer thickness (e.g., $d_{33}^{\text {eff }} \approx-\frac{h}{\gamma d}\left(d_{33}^{S}+(1+2 v) d_{31}^{S}\right)$ for ultrathin films) and the tip position when $d_{i j k}^{S}=d_{i j k}^{S}\left(y_{1}, y_{2}\right)$. It is clear that the value of $d_{33}^{V}$ can be found as the difference between positive and negative piezoresponse saturating values.

For the more complex case of a hardly switchable surface polar layer, the shift $d_{33}^{V}$ described by Eq. (5) is voltage-independent only at $U \leq U_{S}$ and should be distinguished from the aforementioned downward loop shift $d_{33}^{D}$. At $U>U_{S}$, the surface layer can switch and the piezoresponse loop can become almost symmetric. It is clear that the value of $d_{33}^{V}$ can be found as the

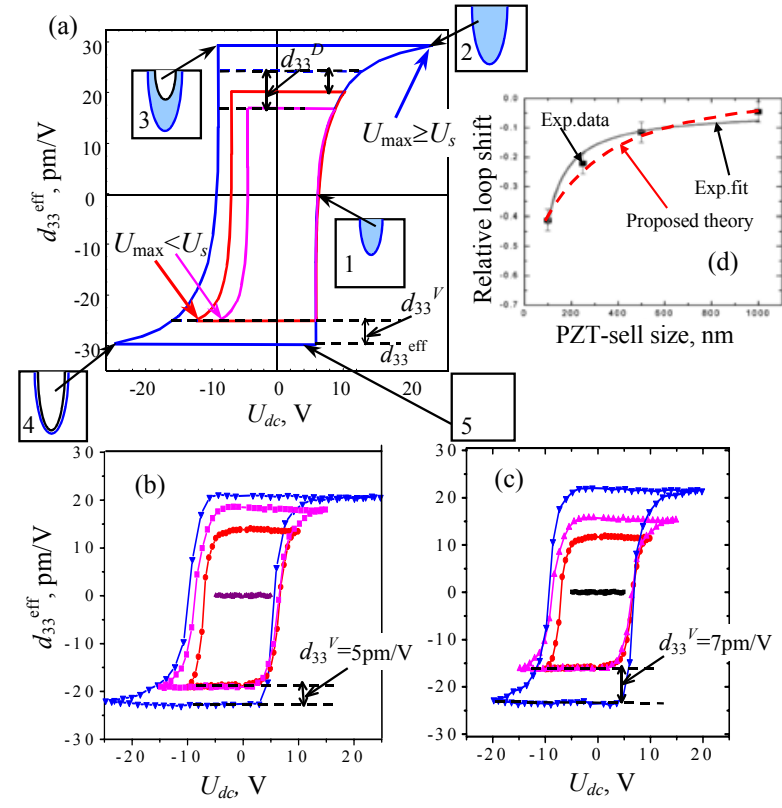

Fig. 5. Piezoresponse loops at different maximal biases $U_{\max }$ (a) - results of theoretical calculations in the kinetic limit; (b,c) - experiment for a multiferroic $\mathrm{BiFeO}_{3}$ film from Ref. [17]. (d) -size dependence of the relative hysteresis loop shift (imprint) in mesoscopic PZT cells; symbols and solid curve - experimental data and their empirical fit from Ref. [3], dashed curve - results of our theoretical calculations of the relative response $d_{33}^{V}(h) / d_{33}^{\text {eff }}\left(h_{0}-h\right) \quad(h$ is the nonswitchable surface layer thickness, and $h_{0}$ is the cell thickness).

difference between negative piezoresponse saturating values: $d_{33}^{V}=d_{33}^{\text {eff }}\left(U>>U_{S}\right)-d_{33}^{\text {eff }}\left(U<U_{S}\right)$. Such an example is schematically shown in Fig. 5a. Relevant experimental loops are shown in Fig. 5b, c. The size dependence of the relative hysteresis loop shift (imprint) is shown in Fig. 5 d.

\section{Conclusion}

Piezoresponse size effects can be extrinsic (as considered in the present paper) or intrinsic ones (e.g., related to the inhomogeneous polarization distribution in nanostructures). If the values of critical thickness $h_{\mathrm{cr}}$ for the intrinsic size-induced paraelectric phase transition and the effective charge-surface separation $d$ are of the same order, extrinsic and intrinsic size effects should interfere for realistic experiments in thin films. Their contributions can be separated by fitting the experimental data provided by the proposed analytical expressions accompanied with the appropriate "intrinsic" dependence of the piezoelectric coefficient 
$d_{i j}^{S}(h)$. Appropriate theoretical calculations are in quantitative agreement with the piezoresponse of a $\mathrm{PbZr}_{0.52} \mathrm{Ti}_{0.48} \mathrm{O}_{3}$ film on $\mathrm{SrTiO}_{3}$ substrate. Thus, the obtained simple Pade approximations and the exact series can be applied to calculations of the local piezoelectric response of polar layers capped on the nonpiezoelectric bulk.

We have proposed an analytical expression for the local vertical shift $d_{33}^{V}$ of effective piezoelectric response hysteresis loops caused by the surface piezoeffect $d_{i j k}^{S}$. The theoretical result is in qualitative agreement with the asymmetry of typical piezoresponse loops obtained for multiferroic $\mathrm{BiFeO}_{3}$ and mesoscopic PZT cells.

\section{References}

1. A.K. Tagantsev, Piezoelectricity and flexoelectricity in crystalline dielectrics // Phys. Rev. B 34, p. 5883 (1986).

2. Y. Saya, S. Watanabe, M. Kawai, H. Yamada, K. Matsushige, Investigation of nonswitching regions in ferroelectric thin films using scanning force microscopy // Jpn J. Appl. Phys. 39, p. 3799 (2000).

3. M. Alexe, C. Harnagea, D. Hesse, U. Gosele, Polarization imprint and size effects in mesoscopic ferroelectric structures // Appl. Phys. Lett. 79, p. 242 (2001).

4. I. Szafraniak, C. Harnagea, R. Scholz, et al., Ferroelectric epitaxial nanocrystals obtained by a self-patterning method // App. Phys. Lett. 83, p. 2211 (2003).

5. W. Ma, and D. Hesse, Polarization imprint in ordered arrays of epitaxial ferroelectric nanostructures // App. Phys. Lett. 84, p. 2871 (2004).

6. A.N. Morozovska, E.A. Eliseev, S.L. Bravina, and S.V. Kalinin, Resolution function theory in piezoresponse force microscopy: domain wall profile, spatial resolution, and tip calibration // Phys. Rev. B. 75(17), 174109-1-18 (2007).

7. F. Felten, G.A. Schneider, J.M. Saldaña, and S.V. Kalinin, Modeling and measurement of surface displacements in $\mathrm{BaTiO}_{3}$ bulk material in piezoresponse force microscopy // J. Appl. Phys. 96, p. 563 (2004).

8. D.A. Scrymgeour and V. Gopalan, Nanoscale piezoelectric response across a single antiparallel ferroelectric domain wall // Phys. Rev. B 72, 024103 (2005).

9. A. N. Morozovska, E.A. Eliseev, and S.V. Kalinin, The piezoelectric surface layers recognition by piezoresponse force microscopy // J. Appl. Phys. 102(7), 074105-1-12 (2007).

10. A. N. Morozovska, E.A. Eliseev, and S.V. Kalinin, Domain nucleation and hysteresis loop shape in piezoresponse force spectroscopy // Appl. Phys. Lett. 89, 192901 (2006).

11. A.N. Morozovska, S.V. Kalinin, E.A. Eliseev, and S.V. Svechnikov, Polarization screening effect on local polarization switching mechanism and hysteresis loop measurements in piezoresponse force microscopy // Ferroelectrics 354, p.198-207(2007).

12. A.N. Morozovska, S.V. Svechnikov, E.A. Eliseev, and S.V. Kalinin, Extrinsic size effect in piezoresponse force microscopy of thin films // Phys. Rev. B 76(5), 054123-1-5 (2007).

13. N.A. Pertsev, A.G. Zembilgotov, and A.K. Tagantsev, Effect of mechanical boundary conditions on phase diagrams of epitaxial ferroelectric thin films // Phys. Rev. Lett. 80, p.1988 (1998).

14. V. Nagarajan, J. Junquera, J.Q. He, C.L. Jia, R. Waser, K. Lee, Y.K. Kim, S. Baik, T. Zhao, R. Ramesh, Ph. Ghosez, and K.M. Rabe, Scaling of structure and electrical properties in ultrathin epitaxial ferroelectric heterostructures // J. Appl. Phys. 100, 051609 (2006).

15. C. Lichtensteiger, J.-M. Triscone, Javier Junquera and $\mathrm{Ph}$. Ghosez, Ferroelectricity and tetragonality in ultrathin PbTiO3 films // Phys. Rev. Lett. 94, 047603 (2005).

16. D.D. Fong, G.B. Stephenson, S.K. Streiffer, J.A. Eastman, O. Auciello, P.H. Fuoss, and C. Thompson, Ferroelectricity in ultrathin perovskite films // Science 304, p. 1650 (2004).

17. A.N. Morozovska, E.A. Eliseev, S.V. Svechnikov, V. Gopalan, S.V. Kalinin // E-print arxiv: 08014086. 